\title{
PREPARATION OF (BENZO)ISOQUINOLINES USING in situ GENERATED ARYNE INTERMEDIATES
}

\author{
D. S. Kopchuk ${ }^{1,2}$, G. V. Zyryanov ${ }^{1,2, *}$, I. S. Kovalev ${ }^{1}$, I. N. Egorov ${ }^{1}$, \\ V. L. Rusinov ${ }^{1,2}$, and O. N. Chupakhin ${ }^{1,2}$
}

Keywords: aryne intermediates, (benzo)isoquinoline, 1,2,4-triazine, cycloaddition, Diels-Alder reaction.

(Benzo)isoquinoline derivatives are of significant interest in different areas of basic and applied organic chemistry. Of special promise are their biological [1-3], photophysical, and coordination [4-8] properties. Their use as chiral catalysts $[9,10]$ is also of interest.

The most frequently used methods for preparing (benzo)isoquinoline systems are cross-coupling reactions [11, 12], which demand the use of special catalysts. We propose a method for preparing (benzo)isoquinoline derivatives based on an aza Diels-Alder reaction with 1,2,4-triazines as dienes and the aryne intermediates acting as dienophiles.

The methodology for preparing diverse pyridine derivatives via reaction of the corresponding 1,2,4-triazines has long been known [13-15]. However, there are only a few examples of using aryne intermediates in similar reactions. The range of dienophiles in these cases is limited to certain dehydrobenzene derivatives, the generation of which used poorly available anthranilic acids $[16,17]$ despite the marked growth in the chemistry of arynes in recent years $[18,19]$.

In our work, we propose a highly efficient method for the preparation of (het)aryl-substituted isoquinoline 1a and benzo[ $h]$ isoquinoline $\mathbf{1 b}$, which involves the reaction of arynes $\mathbf{2 a}, \mathbf{b}$ with the substituted 1,2,4-triazines 3a,b in anhydrous toluene for $24-36 \mathrm{~h}$ at $140^{\circ} \mathrm{C}$ under an argon atmosphere. The target compounds 1a,b were obtained in $76 \%$ and $80 \%$ yield, respectively. The corresponding arynes were generated in situ by the action of potassium tert-butoxide on the chloro- and bromo-substituted arenes $\mathbf{4 a}, \mathbf{b}$.

The structure of the obtained compounds $\mathbf{1 a}, \mathbf{b}$ was confirmed from the ${ }^{1} \mathrm{H}$ and ${ }^{13} \mathrm{C}$ NMR spectroscopy, mass spectrometry, and by elemental analysis data. The mass spectra of compounds $\mathbf{1 a}, \mathbf{b}$ showed the presence of a molecular ion peak.

The compounds obtained are 2,2'-bipyridine type ligands with an extended conjugated system, and are of interest from the viewpoint of complex formation and as luminescent materials.

\footnotetext{
*To whom correspondence should be addressed, e-mail: gvzyryanov@gmail.com.

${ }^{1}$ Ural Federal University named after the First President of Russia B. N. Yeltsin, 19 Mira St., Yekaterinburg 620002, Russia.

${ }^{2}$ I. Ya. Postovskii Institute of Organic Synthesis, Ural Branch, Russian Academy of Sciences, 22 Akademicheskaya St./20 S. Kovalevskoi St., Yekaterinburg 620219, Russia; e-mail: chupakhin@ios.uran.ru.
}

Translated from Khimiya Geterotsiklicheskikh Soedinenii, No. 12, pp. 1994-1997, December, 2012. Original article submitted February 15, 2012. 

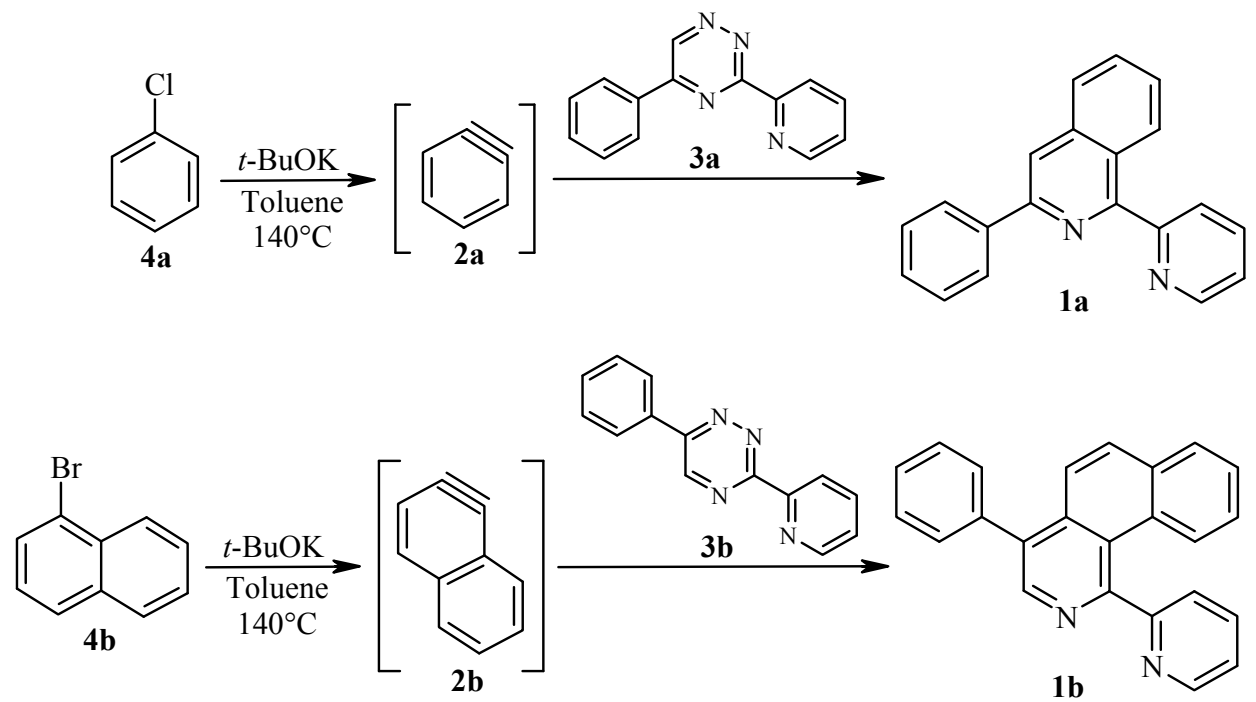

${ }^{1} \mathrm{H}$ and ${ }^{13} \mathrm{C}$ NMR spectra were recorded on a Bruker DRX-400 spectrometer (400 and $100 \mathrm{MHz}$, respectively) using $\mathrm{CDCl}_{3}$ with TMS as internal standard. ESI mass spectra were recorded on a Bruker Daltonics micrOTOF-Q II mass spectrometer. Elemental analysis was conducted on a Perkin-Elmer PE 2400 series II CHN-analyzer. Melting points were determined on a Boetius hot stage apparatus. TLC analysis was performed on Merck 60 F254 silica gel and visualized in UV light. All of the synthetic procedures were carried out under an argon atmosphere.

The 5-phenyl-3-(2-pyridyl)-1,2,4-triazine (3a) [20] and 6-phenyl-3-(2-pyridyl)-1,2,4-triazine (3b) [15] were synthesized by the reported methods.

Preparation of the Aryne Cycloaddition Products (General Method). A suspension of the triazine 3a,b $(0.50 \mathrm{mmol})$, the haloarene $4 \mathbf{a}, \mathbf{b}(0.50 \mathrm{mmol})$, and $t$-BuOK $(85 \mathrm{mg}, 0.75 \mathrm{mmol})$ in absolute toluene $(50 \mathrm{ml})$ was stirred in an autoclave at $140^{\circ} \mathrm{C}$ for $24 \mathrm{~h}$. After cooling to room temperature, the reaction mixture was washed with water $(2 \times 50 \mathrm{ml})$, and the organic fraction was dried over anhydrous $\mathrm{Na}_{2} \mathrm{SO}_{4}$. Toluene was evaporated under reduced pressure, and the residue was purified by column chromatography using $\mathrm{CH}_{2} \mathrm{Cl}_{2}$ as eluent.

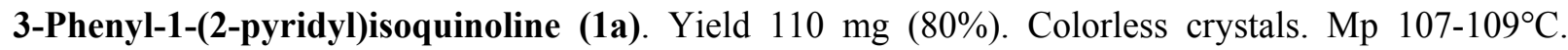
${ }^{1} \mathrm{H}$ NMR spectrum, $\delta$, ppm $(J, \mathrm{~Hz}): 7.41-7.45(3 \mathrm{H}, \mathrm{m}, \mathrm{H} \mathrm{Ph}) ; 7.51-7.53$ (2H, m, H isoquinoline); 7.57-7.59 (1H, $\mathrm{m}, \mathrm{H}$ isoquinoline); 7.68-7.70 $(1 \mathrm{H}, \mathrm{m}, \mathrm{H}$ isoquinoline); 7.93-7.95 $(2 \mathrm{H}, \mathrm{m}, \mathrm{H} \mathrm{Ph}) ; 8.15(1 \mathrm{H}, \mathrm{s}, \mathrm{H}-4) ; 8.21-8.23$ $\left(2 \mathrm{H}, \mathrm{m}, \mathrm{H}\right.$ Py); $8.75\left(1 \mathrm{H}, \mathrm{dd},{ }^{3} J=8.4,{ }^{4} \mathrm{~J}=1.8, \mathrm{H}-3 \mathrm{Py}\right) ; 8.81\left(1 \mathrm{H}, \mathrm{dd},{ }^{3} J=4.8,{ }^{4} J=1.8, \mathrm{H}-6\right.$ Py $) .{ }^{13} \mathrm{C}$ NMR spectrum, $\delta$, ppm: $117.0 ; 123.2 ; 125.6 ; 127.0 ; 127.4 ; 127.5 ; 128.0 ; 128.5 ; 128.6 ; 128.8 ; 130.1 ; 137.0 ; 138.2$; $139.5 ; 148.3 ; 149.8 ; 157.0 ; 158.8$. Mass spectrum, $m / z\left(I_{\text {rel }}, \%\right): 283.12[\mathrm{M}+\mathrm{H}]^{+}(100)$. Found, \%: C 84.81; $\mathrm{H} 4.80 ; \mathrm{N} 9.61 . \mathrm{C}_{20} \mathrm{H}_{14} \mathrm{~N}_{2}$. Calculated, \%: C 85.08; H 5.00; N 9.92.

4-phenyl-1-(2-pyridyl)benzo $[\boldsymbol{h}]$ isoquinoline (1b). Yield $130 \mathrm{mg}$ (76\%). Colorless crystals. Mp 202-204 ${ }^{\circ} \mathrm{C} .{ }^{1} \mathrm{H}$ NMR spectrum, $\delta$, ppm $(J, \mathrm{~Hz}): 7.29-7.33(2 \mathrm{H}, \mathrm{m}, \mathrm{H}-5 \mathrm{Py}, \mathrm{H} \mathrm{Ar}) ; 7.49-7.64(6 \mathrm{H}, \mathrm{m}, \mathrm{H} \mathrm{Ph}$, $\mathrm{H}$ Ar); 7.45-7.86 (6H, m, H Ph, H Ar); 8.22-8.24 (2H, m, H-3,6 Py). ${ }^{13} \mathrm{C}$ NMR spectrum, $\delta$, ppm: 120.4; 122.9; $124.5 ; 126.9 ; 128.1 ; 128.4 ; 128.9 ; 129.6 ; 130.1 ; 131.2 ; 131.7 ; 133.2 ; 133.9 ; 136.8 ; 137.7 ; 142.9 ; 144.2 ; 149.2$; $149.9 ; 151.6 ; 153.7 ; 160.7$. Mass spectrum, $m / z\left(I_{\text {rel, }} \%\right): 333.15[\mathrm{M}+\mathrm{H}]^{+}(100)$. Found, \%: C 86.55; H 4.76; N 8.21. $\mathrm{C}_{24} \mathrm{H}_{16} \mathrm{~N}_{2}$. Calculated, \%: C 86.72; H 4.85; N 8.43.

This work was carried out with the support of the Ministry of Education and Science of the Russian Federation (state contract Nos. 14.740.11.1020 and 14.A18.21.0817). 


\section{REFERENCES}

1. N. Atatreh, C. Stojkoski, P. Smith, G. W. Booker, C. Dive, A. D. Frenkel, S. Freeman, and R. A. Bryce, Bioorg. Med. Chem. Lett., 18, 1217 (2008).

2. A. Cappelli, G. Giuliani, A. Gallelli, S. Valenti, M. Anzini, L. Mennuni, F. Makovec, A. Cupello, and S. Vomero, Bioorg. Med. Chem., 13, 3455 (2005).

3. D. B. Khadka and W.-J. Cho, Bioorg. Med. Chem., 19, 724 (2011).

4. H. Matsumiya, H. Hoshino, and T. Yotsuyanagi, Analyst, 126, 2082 (2001).

5. $\quad$ P.-T. Chou and C.-Y. Wei, J. Phys. Chem., 100, 17059 (1996).

6. D. Prema, A. V. Wiznycia, B. M. T. Scott, J. Hilborn, J. Desper, and C. J. Levy, J. Chem. Soc., Dalton Trans., 4788 (2007).

7. M. Bardaji, A. B. Miguel-Coello, and P. Espinet, Inorg. Chim. Acta, 392, 91 (2012).

8. $\quad$ B. Liang, C. Ouyang, Z. Chen, H. Zhu, and Z. Xiao, Adv. Sci. Lett., 10, 253 (2012).

9. C. W. Lim, O. Tissot, A. Mattison, M. W. Hooper, J. M. Brown, A. R. Cowley, D. I. Hulmes, and A. J. Blacker, Org. Process Res. Dev., 7, 379 (2003).

10. B. A. Sweetman, H. Muller-Bunz, and P. J. Guiry, Tetrahedron Lett., 46, 4643 (2005).

11. C. Rochais, R. Yougnia, T. Cailly, J. Sopkova-de Oliveira Santos, S. Rault, and P. Dallemagne, Tetrahedron, 67, 5806 (2011).

12. D. M. Knapp, E. P. Gillis, and M. D. Burke, J. Am. Chem. Soc., 131, 6961 (2009).

13. G. R. Pabst, O. C. Pfüller, and J. Sauer, Tetrahedron, 55, 8045 (1999).

14. A. Rykowski, D. Branowska, and J. Kielak, Tetrahedron Lett., 41, 3657 (2000).

15. V. N. Kozhevnikov, O. V. Shabunina, D. S. Kopchuk, M. M. Ustinova, B. König, and D. N. Kozhevnikov, Tetrahedron, 64, 8963 (2008).

16. A. M. d'A. R. Gonsales, M. V. D. Pinho e Melo, and T. L. Gilchrist, Tetrahedron, 48, 6821 (1992).

17. S. Diring, P. Retailleau, and R. Ziessel, Tetrahedron Lett., 48, 8069 (2007).

18. P. M. Tadross and B. M. Stoltz, Chem. Rev., 112, 3550 (2012).

19. I. S. Kovalev, D. S. Kopchuk, G. V. Zyryanov, P. A. Slepukhin, V. L. Rusinov, and O. N. Chupakhin, Khim. Geterotsikl. Soedin., 576 (2012). [Chem. Heterocycl. Compd., 48, 536 (2012)].

20. M. O'Rourke, S. A. Lang, Jr., and E. Cohen, J. Med. Chem., 20, 723 (1977). 